\title{
Análisis de la transmisión de energía eléctrica en un sistema hexafásico
}

\section{Analysis of the transmission of electric power in a hexaphasic system}

\author{
aCastañeda Quinte, M.; Rodríguez García, E.; Colonio Llacua, J. \\ Facultad de Ingeniería Eléctrica y Electrónica / Universidad Nacional del Centro del Perú \\ Email: jcolonio@uncp.edu.pe
}

\section{Resumen}

El consumo de energía en el transcurso del tiempo está aumentando, ello lleva a aumentar la capacidad de potencia a transmitir, elevando los niveles de tensión: HV (High Voltaje), Extra EHV (High Voltaje) y UHV (Ultra High Voltaje); recorriendo, muchas veces, al uso de doble terna con $1,2,3 \ldots$ conductores por fase. Al aumentar el nivel de tensión de transmisión, se está aumentando: nivel de aislamiento, dimensionamiento de las torres, franja de servidumbre, pérdidas por efecto corona, campos electromagnéticos, etc. Otra alternativa sería la transmisión de la energía eléctrica hexafásica.

Mediante el modelado del sistema $3 \Phi$ doble terna y $6 \Phi$ en MATLAB, se ha demostrado que los sistemas hexafásicos presentan una mejor estabilidad, comparadas con la transmisión trifásica doble terna en la capacidad de transmisión de potencia y caídas de tensión. Además de que una línea hexafásica puede llevar hasta $73 \%$ más de potencia eléctrica que una doble terna trifásica, para las mismas dimensiones de la faja de servidumbre.

Palabras clave: transmisión de energía, sistema hexafásico, corriente trifásica, modelado en Matlab

\begin{abstract}
The energy consumption in the course of time is increasing, this leads us to increase the capacity of power to transmit, increasing voltage levels: HV (High voltage), Extra EHV (High voltage) and UHV (Ultra High voltage); Traveling many times to the use of double terna with 1,2, 3... Drivers per phase. By increasing the level of transmission voltage, it's increasing: level of insulation, dimensioning of the towers, band of servitude, lost by corona effect, electromagnetic fields, etc. Another alternative would be the transmission of hexaphasic electric power.

By modeling the system $3 \Phi$ double terna and $6 \Phi$ in MATLAB, it has been shown that the six phase systems have a better stability, compared with the three-phase double-triple transmission in the capacity of power transmission and voltage drops. Besides that a hexaphasic line can carry up to $73 \%$ more of electric power than a double three-phase, for the same dimensions of the band of servitude.
\end{abstract}

Keywords: power transmission, hexaphasic system, triphasic current, modeling in Matlab 


\section{Introducción}

El proyecto de investigación se orienta a la comprensión, modelado y simulación de un sistema de transmisión hexafásico, cuyos componentes son: generador, transformador y línea de transmisión. Con el presente estudio, se propone una solución a la limitada capacidad de transmisión de la energía eléctrica de las líneas de transmisión existentes; asimismo, obtener múltiples ventajas respecto al sistema trifásico. A continuación, se describe brevemente el contenido de cada capítulo:

El capítulo I - Marco teórico: describe las tensiones y las relaciones fasoriales, tanto para el sistema trifásico como para el hexafásico. También, se establece el modelado del sistema hexafásico.

El capítulo II - Materiales y métodos: describe los materiales y metodología que se utilizaron para cumplir con los objetivos propuestos en el plan del proyecto de investigación.

El capítulo III - Resultados: se realiza el análisis del modelado en MATLAB del sistema $3 \phi$ doble terna y $6 \phi$; además, se analiza el comportamiento del campo electromagnético para el sistema $3 \phi$ doble terna y $6 \phi$; finalmente, se describen las ventajas del sistema $6 \phi$ frente al $3 \phi$.

El capítulo IV - Discusiones: se argumentan diversas modificaciones del sistema $6 \phi$. Al final, se describe un análisis de costos del sistema $6 \phi$.

\section{Métodos y materiales}

El método utilizado fue analítico, ya que se modeló el sistema de transmisión de la energía eléctrica hexafásica y, se realizó un estudio de los distintos elementos que lo componen.

El nivel fue explicativo no experimental, por explicar el comportamiento de la transmisión de energía en un sistema hexafásico. El tipo de investigación fue básica.

\section{Población y muestra}

La población fue el sistema eléctrico hexafásico. Se recolectaron datos y se registraron en Excel MS; así como, para la programación correspondiente de cada modelo matemático.

\section{Materiales}

Se utilizaron estudios, informes y software. Se emplearon programas computaciones para procesar los datos.

\section{Resultados}

Se realizó el modelado en MATLAB, para las siguientes conexiones:

\section{Y-Y y $Y$ - $Y$ invertida}

- Esta configuración no puede utilizarse para la mejora de la capacidad de transmisión de potencia en las líneas, por que los resultados de simulación muestran que, en este caso, la tensión de fase no se aumenta a tensiones de línea, pero la tensión de línea se reduce a tensiones de fase.

- Comparando los gráficos, con los del sistema trifásico, se puede concluir que la magnitud de voltaje de la línea se reduce a la tensión de fase en el caso del hexafásico.

- Los gráficos, también indican claramente que la magnitud de las tensiones de fase es aproximada, igual a tensiones de línea y de la diferencia de fase entre dos fases consecutivas es de $60^{\circ}$.

\section{$\Delta-Y$ y $\Delta$ - $Y$ invertida}

- En esta configuración, el voltaje de fase se incrementa a la tensión de línea en contraste con la conexión Y-Y y $Y$ - $Y$ invertida, configuración donde el voltaje de línea se reduce a la tensión de fase.

- Si se examina cuidadosamente los gráficos correspondientes, sería evidente que las tensiones de fase se han incrementado y aproximado a las tensiones de línea. La magnitud de la tensión de línea es igual a la tensión de fase, pero el voltaje de línea adelanta a la de fase en $60^{\circ}$.

- Esta conexión mejora la capacidad de transmisión de potencia en las líneas, en $\sqrt{3}$, o un $73 \%$ extra de capacidad de transmisión. Por lo tanto, para la misma corriente de alimentación de la línea de carga en serie RLC se multiplica por 1,73; es decir, la potencia, activa, inductivos y capacitivos se multiplican por un factor de 1,73 .

\section{Conexión diametral}

Los resultados de configuraciones diametrales son exactamente igual que el de las configuraciones $\Delta$-Y y $\Delta$-Y invertida, como se ven en las figuras 1 , 2 y 3.

\section{Discusión}

El sistema de transmisión $6 \Phi$ ofrece la oportunidad de responder a las crecientes demandas de energía y, al mismo tiempo, cumplir con las restricciones ambientales y de regulación. Sin embargo, los factores de la economía tienen que ser considerados como gastos de salida que pueden ser muy altos para las líneas $6 \Phi$. Una línea $6 \Phi$ requeriría transformadores de conversión que causan en los terminales un mayor costo. El alto coste de los terminales, se ve compensado por la reducción de la torre y reducción de los costes de cimentación, el costo de franja de servidumbre y pérdidas. 


\section{Figura 1}

Campo magnético para la línea de transmisión $6 Ф$

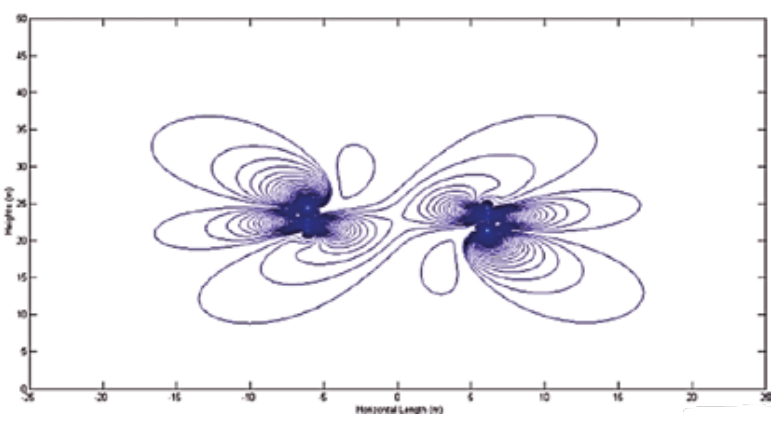

Figura 2

Campo magnético para la línea de transmisión con un $73 \%$ de carga adicional

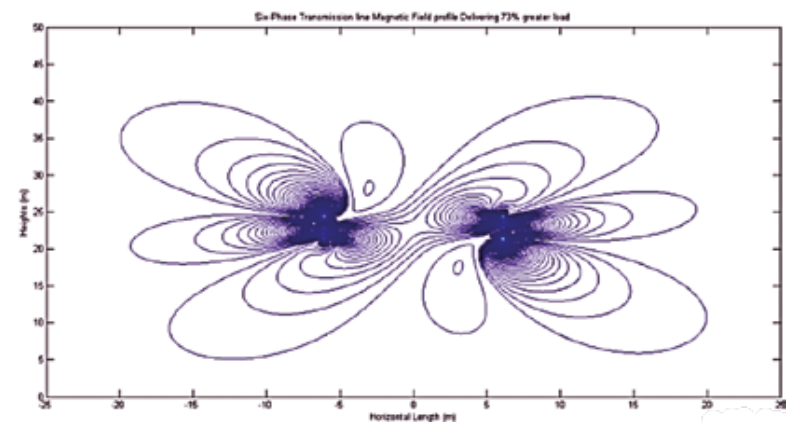

Figura 3

Perfil del campo magnético para la línea de transmisión $6 Ф$

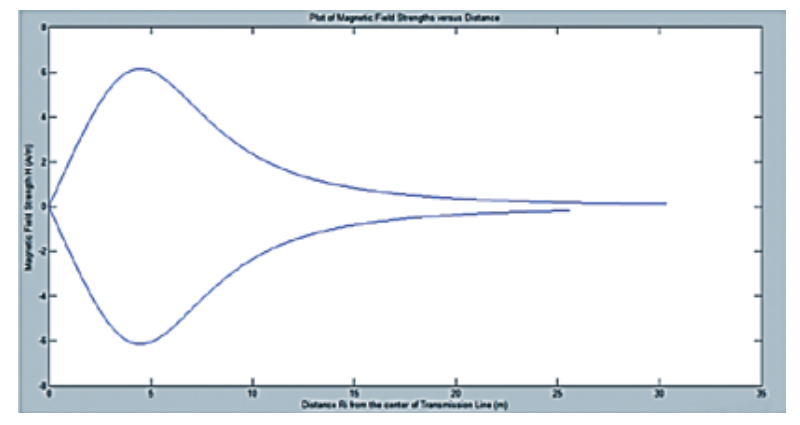

\section{Conclusiones}

- Entre los sistemas High Phase Order (HPO) o sistemas multifásicos, la transmisión $6 \Phi$ se prueba que es más fiable para incrementar la capacidad de las líneas de transmisión existentes y, al mismo, proporciona: menor utilización de la franja de servidumbre, mayor capacidad de transferencia de potencia, estructura más pequeña, menor nivel de aislamiento, mayor estabilidad en el sistema, bajo efecto corona y campos electromagnéticos, aumento de la confiabilidad, reducción del campo electromagnético, entre otros.
- Mediante el modelado del sistema $3 \Phi$ doble terna y 6Ф en MATLAB, se ha demostrado que los sistemas hexafásicos presentan una mejor estabilidad, comparadas con la transmisión trifásica doble terna en la capacidad de transmisión de potencia y caídas de tensión. Además, de que una línea hexafásica puede llevar hasta $73 \%$ más de potencia eléctrica que una doble terna-trifásica, para las mismas dimensiones de la faja de servidumbre.

- En el análisis del campo electromagnético, para el sistema $3 \Phi$ doble terna y $6 \Phi$, se concluye: la intensidad de campo magnético entre los conductores de la línea de $6 \Phi$ es menor que la línea de transmisión $3 \Phi$ doble terna, por lo que se requiere menos espacio conductor en los conductores de seis fases. Además, el campo magnético de la de la línea de transmisión de $6 \Phi$, sostiene un valor muy pequeño, incluso después de 20 metros de distancia, desde el centro de la línea, pero el valor es inferior a $2 \mathrm{~A} / \mathrm{m}$ que es ambientalmente seguro. Así, la línea de transmisión de transmisión de $6 \Phi$, no tendrá ningún problema en viabilidad respecto a las preocupaciones de campo magnético, pero tiene una ventaja, que las estructuras compactas pueden hacer que requieran menos espaciamiento del conductor.

- Los factores de la economía tienen que ser considerados, como los gastos de salida que pueden ser muy altos para las líneas $6 \Phi$; el costo de implementación del sistema $6 \Phi$, será compensado por las múltiples ventajas que ofrece.

\section{Recomendación}

- En este proyecto, la simulación de línea de transmisión eléctrica de $6 \Phi$ se realiza en MATLAB y Simulink SimPowerSystems. La precisión de MATLAB es limitada, en el futuro para obtener resultados más precisos se recomienda utilizar software como PSCAD, PSSE etc. La investigación debe hacerse a nivel práctico por las compañías de transmisión mediante la implementación de hardware en una larga longitud de línea de transmisión.

\section{Referencias bibliográficas}

Xianda Deng. (2012) Exploring Six-Phase transmission lines for increasing power transfer with limited right of way. Arizona State University. December.

Joseph, E. and Fleckenstein. (2016) Three-Phase electrical power. Edit.CRC Press, United States. 